\title{
Impairment of cell division of Trypanosoma cruzi epimastigotes
}

\section{Michele A Zacks}

Department of Pathology, University of Texas Medical Branch, 301 University Boulevard, Galveston, TX 77555-0609, US

The mechanisms that facilitate the adaptation of Trypanosoma cruzi to two distinct hosts, insect and vertebrate, are poorly understood, in part due to the limited ability to perform gene disruption studies by homologous recombination. This report describes a developmentally-defective phenotype that resulted from integration of a drug marker adjacent to the GAPDH gene in T. cruzi.

Key words: Trypanosoma cruzi - life cycle - differentiation

Trypanosoma cruzi is a protozoan parasite of the ancient branch of eukaryotes (Kingdom Eukaryota, Order Kinetoplastida) (Stevens et al. 1999) and is endemic in South and Central America, and Mexico. T. cruzi is transmitted to vertebrates, including humans, predominantly by insect vector (subfamily Triatoma, family Reduviidae) and also occurs via blood transfusion, organ transplantation, and congenital routes. In humans, $30 \%$ of chronically infected individuals are estimated to develop Chagas disease, a distinct form of cardiomyopathy (Miles 2003). Despite the description of the association between $T$. cruzi transmission via the triatomine insect vector and heart disease in 1909 and decades of research illuminating the extraordinary mechanism of host cell infection (Hall 1993, Burleigh \& Andrews 1998), no vaccine is available and only two anti-parasitic drugs have been licensed for treatment. However, these drugs are effective mainly at the acute stage of infection and are highly toxic (Barrett et al. 2003). The mechanisms that facilitate the adaptation of T. cruzi to two distinct hosts, insect and vertebrate, are poorly understood, in part due to the limited ability to perform gene disruption studies by homologous recombination. This report describes a developmentally-defective phenotype that resulted from integration of a drug marker adjacent to the GAPDH gene in T. cruzi. This study was initiated to evaluate the role of surface expressed glycosylphophatidylinositol (GPI)-anchored proteins in the complex life cycle of $T$. cruzi and utilized a homologous recombination-mediated approach to targeted disruption of the TcGPI8 gene in the parasite, described in Zacks and Garg (2006) (Fig. 1). Elec-

Financial support: American Heart Association, John Sealy Memorial Endowment Fund for Biomedical Research, American Health Assistance Foundation, the National Institutes of Health (AI053098-01, AI054578-01), the James W. McLaughlin Predoctoral Fellowship Fund, the Department of Pathology and the Graduate School of Biomedical Sciences at the University of Texas Medical Branch

E-mail:mazacks@utmb.edu.

Received 18 May 2006

Accepted 29 November 2006 troporation with a neomycin-resistance (neo ${ }^{r}$ )-based construct targeting TcGPI8 and subsequent drug selection of transfectants resulted in genomic integration of the $n e o^{r}$ cassette, as confirmed by Southern blot analysis (Fig. 1) and PCR amplification of the 1.3 kilobase GAPDH-IR-neo ${ }^{r}$ fragment from gDNA of the transfectants (data not shown). However, GAPDHIR-neo ${ }^{r}$ did not integrate into the TcGPI8 gene and the $3^{\prime}$ and 5 ' ends of TcGPI8 were absent (data not shown). Genome walking and PCR-cloning identified the site of insertion of the GAPDHIR/neo ${ }^{r}$ cassette (Fig. 2A-B) as adjacent to

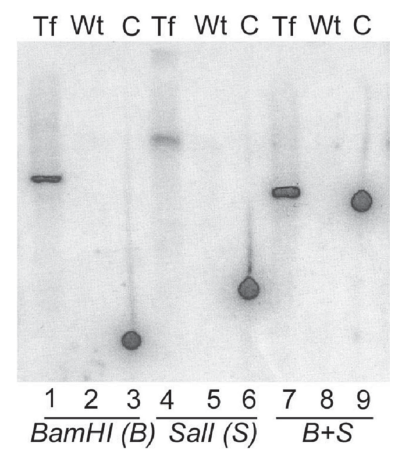

Fig. 1: genetic analysis of stable transfectants. Constructs for disruption of TcGPI8 were designed such that drug resistance genes, neo ${ }^{r}$ or $b l e^{r}$, were flanked by 400-600 base pairs of the 5' and 3' ends of the TcGPI8 gene. The GAPDH-intergenic region (GAPDH-IR) sequence, present upstream of the neo ${ }^{r}$ or $b l e^{r}$ genes, provides the necessary splice acceptor site for mRNA processing (Nozaki \& Cross 1995). Trypanosoma cruzi SylvioX10/4 strain (American Type Culture Collection, Manassas, VA) epimastigotes were electroporated with the linearized disruption cassette $(10 \mu \mathrm{g})$ and incubated in selective drug $(\mathrm{G} 418,60 \mu \mathrm{g} / \mathrm{ml})$ (Kelly et al. 1992). Following positive selection, as evidenced by the death of mocktransfected parasites cultured at the same drug concentration, the drug pressure for selection of transfectants was increased to $200-400 \mu \mathrm{g} / \mathrm{ml}$; after greater than one month, chromosomal DNA (gDNA) was extracted from these stable transfectants (Medina-Acosta \& Cross 1993). For Southern blot analysis, gDNA isolated from stable transfectants or from wild type, untransfected parasites was digested with restriction enzymes (BamHI and/or SalI) and resolved via agarose gel electrophoresis. Following DNA transfer to nylon membrane, hybridization was performed using a ${ }^{32} \mathrm{P}$-labeled neo ${ }^{r}$ probe (random primer labeling) at $68^{\circ} \mathrm{C}$. After 6 days exposure, the image was scanned by phosphorimager. gDNA from stable transfectants hybridized with the neo ${ }^{r}$ probe (lanes 1,4 , and 7) indicating that neo ${ }^{r}$ is present in the transfectant genome. As expected, no hybridization with the neo ${ }^{r}$ probe was detected for wild type, untransfected gDNA (lanes 2, 5, and 8). Bands were detected at the predicted size for plasmid ( $\left.n e o^{r}-T c G P I 8\right)$ DNA (positive control, lanes 3 , 6 , and 9). 


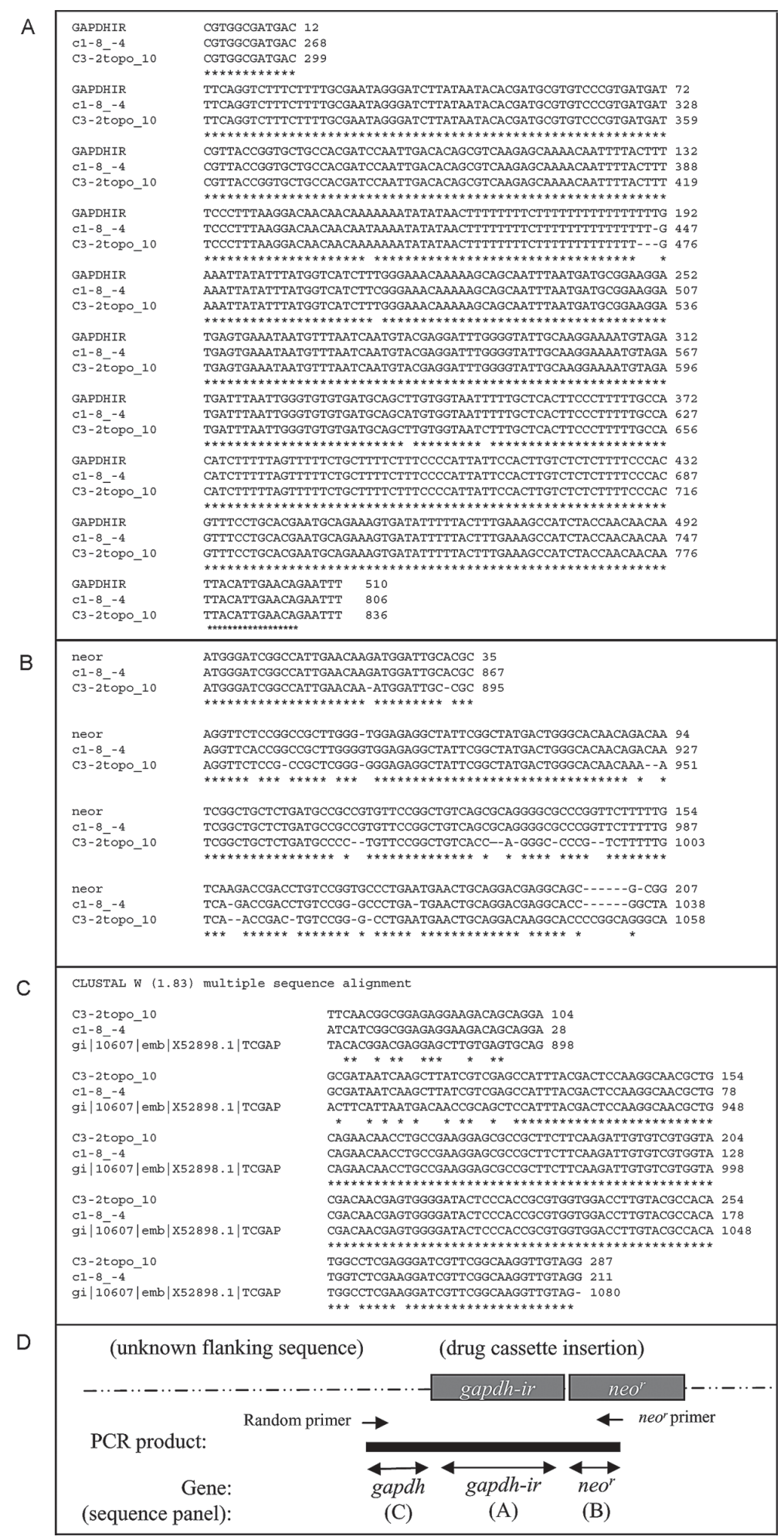

Fig. 2: identification of the site of integration of GAPDHIR-neo ${ }^{r}$ cassette in Trypanosoma cruzi genome. Genome walking via an arbitrarily primed PCR approach was used to determine the site of integration (Parker et al. 1991, Liu \& Whittier 1995). Briefly, random, degenerate primers (Liu \& Whittier 1995) were used in pairs with $n e o^{r}$-specific primers (nested primers R1, 5'-TTTCGCTTGGTGGTCGAATGGGCAGGTA-3'; R2, 5'GCACAGCTGCGCAAGGAACGCCC-3': R3, 5'-GCCGCGCTGCCTCG-3') to amplify fragments from gDNA of stable neo ${ }^{r}$-Tc GPI8 transfectants containing the unknown sequence flanking GAPDHIR-neo ${ }^{r}$ using modified cycling parameters (Liu \& Whittier 1995). PCR fragments were cloned and sequenced (UTMB Protein Core Facility). Three informative clones were obtained, e.g., clones in which the DNA sequence matched to the expected GAPDHIR, and $n e o^{r}$ portions and in which additional flanking sequence was present. T. cruzi blast search was performed using these flanking sequences. The sequence of the third clone was identical to c1-8_-4. Sequence alignments of TAIL-PCR clones with A) GAPDHIR, B) neo $r$, and C) GAPDH are shown. The GAPDHIR and neo ${ }^{r}$ portions of the disruption cassette were confirmed and $T$. cruzi blast search identified the sequence flanking the 5' end of the GAPDHIR-neor ${ }^{r}$ insertion as matching to the 3 ' end of the T. cruzi GAPDH gene (accession \#X52898) (Kendall et al. 1990). 
the 3' end of GAPDH (Fig. 2C) (Kendall et al. 1990). The orientation of the insertion is shown in Fig. 2D.

The morphology of epimastigote-stage transfectants was documented via confocal microscopy (Fig. 3). Stable $n e o^{r}$-Tc GPI8 transfectants (Fig. 3A) showed unusual morphologies, first with the appearance of thin extended forms that appeared to be reduced in motility. Highly motile parasites with two or three flagella were present with frequent observation ( $>2$ per microscopy field) of two parasites with fused membranes, either in apical or longitudinal style. The nucleic acid staining pattern observed in doublet parasites incubated with the fluorescent Syto11 dye indicated that duplication of kinetoplast and nuclei was not impaired. However, in many cases, cellular membranes appeared to be fused. The possibility that these represent intermediate morphological forms in normal T. cruzi division cannot be excluded. However, such parasites were not found in either routine (e.g. daily) light microscope examination of nontransformed cultures during consideration over an extensive period of cultivation with selective drug (e.g., G418 for minimum of 2-3 months) or without selective drug while maintained in parallel cultures using liver in- fusion tryptose medium. Nor, as presented here, were such forms documented during confocal microscopy. This observation is consistent for this study as well as for cultures of pTEX transformed cultures that were continuously cultured for $>2$ years in the presence of selective drug (e.g., G418, as reported here), under daily to weekly monitoring. At the time this phenotype became pronounced, growth of the transformant population declined and could not be further maintained, most likely as a result of their inability to complete their replication cycle. Thus, at the epimastigote stage, a striking defect in cell division occurred in transfectants.

The effect of this transformation on the life cycle development of transfectants, e.g., differentiation of epimastigote to the infective metacyclic forms and infection of mammalian cells, was further evaluated. Transfectant epimastigotes and, as control, wild type epimastigotes were grown in liver infusion tryptose medium for $\geq 10$ days without addition of media to achieve stationary-growth phase for the conversion of parasites to metacyclic trypomastigotes and were used to infect fibroblast monolayers at a parasite to cell ratio of 50:1. At $24 \mathrm{~h}$ after infection, medium containing free
A
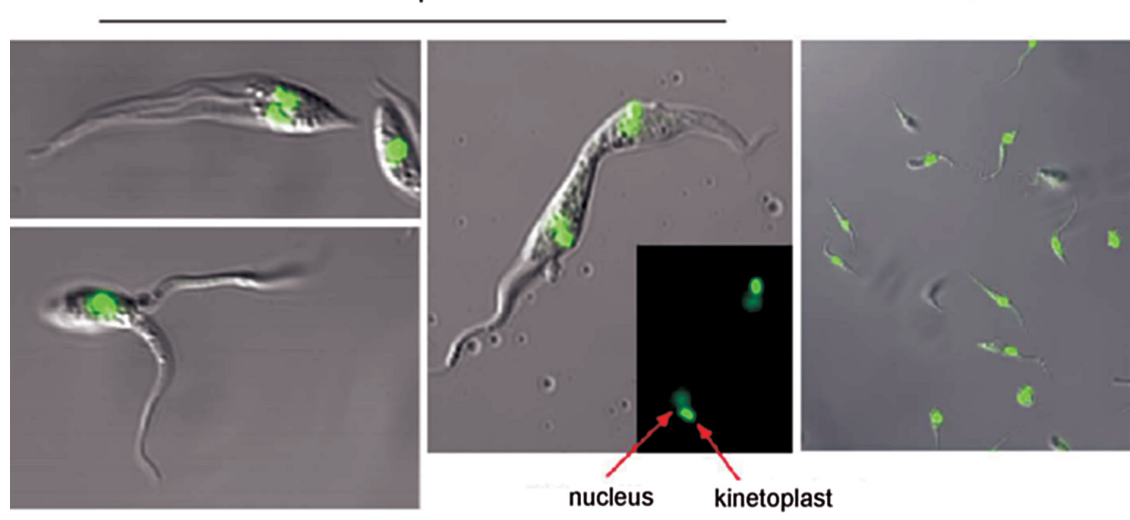

B

i

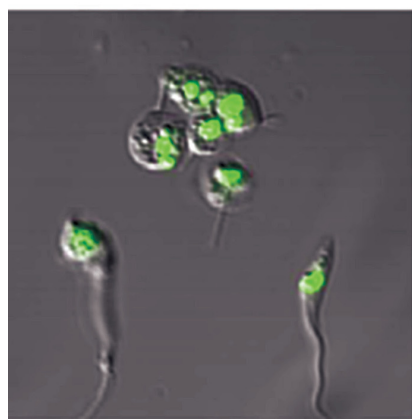

nucleus

ii

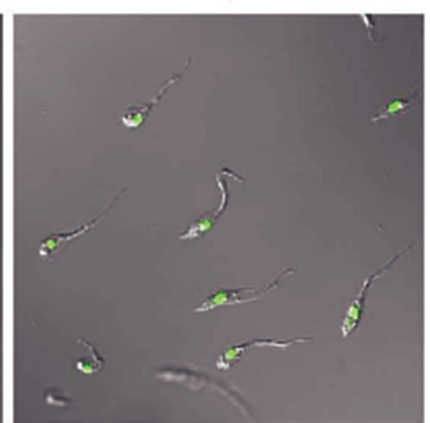

Fig. 3: morphological characteristics of stable transfectants. Stable neo ${ }^{r}$-Tc GPI8 transfectants were generated and genetically characterized, as described in Figs 1 and 2. Parasites were harvested by centrifugation and washed in ice-cold PBS. Epimastigote morphology was documented via confocal microscopy of live parasites. To visualize kinetoplast and nuclear DNA, epimastigotes were incubated on ice for 10 min with Syto11 (Molecular Probes, 1:200 dilution), a cell-permeant nucleic acid binding fluorescent dye (excitation $\lambda_{\text {Max }} 515 \mathrm{~nm}$; emission $\lambda_{\text {Max }}, 543 \mathrm{~nm}$ ). Confocal images were captured on a Zeiss LSM 510 UV Meta Laser Scanning Confocal Microscope (UTMB Optical Imaging Core Facility) at a magnification of $63 X$. Signals were overlaid with Nomarski differential interference using Zeiss AxioVision Viewer software. $A$ : stable $n e o^{r}-T c G P I 8$ transfectants. i: transfectants; ii: wild type untransfected $T$. cruzi. Kinetoplast and nuclear DNA, which replicated in doublet parasites, are indicated by arrow; $B$ : neo ${ }^{r}$ TcGPI8 transfectants following targeting with $b l e^{r}$-TcGPI8. Stable neo ${ }^{r}$-TcGPI8 transfectants were electroporated with a second, ble ${ }^{r}$-based disruption construct and selected in phleomycin, as described in the text. i: transfectants; ii: wild type untransfected T. cruzi. 
parasites was replaced and cells monitored for the appearance of trypomastigotes. Three independent in vitro infection experiments were performed in two different fibroblast cell lines (C2C12 and BHK21) using stationary-phase cultures of stable transfectants or of wild type untransfected T. cruzi. Transfectants did not convert to the typical morphology of the infective metacyclic form following $\geq 10$ days of cultivation without addition of new growth medium. No trypomastigotes were seen when monitoring fibroblasts infected with the stationary phase cultures of these $n e o^{r}$-TcGPI8 transfectants during a 10-12 day incubation period. However, wild type, untransfected parasites were capable of infecting either fibroblast line, with appearance of trypomastigotes and amastigotes within 6 and 11 days, respectively. In summary, morphological transformation of the neor-TcGPI8 transfectant epimastigotes into metacyclic trypomastigotes in culture conditions of "starvation" did not occur, unlike for wild type parasites. In conclusion, these stable $n e o^{r}$-TcGPI8 transfectants were not infective for mammalian cells in vitro.

In effort to obtain homozygous transfectants, the stable neo ${ }^{r}$-TcGPI8 transfectants were subsequently electroporated with a similar disruption construct containing the phleomycin-resistance $\left(\right.$ ble $\left.{ }^{r}\right)$ gene in place of the $n e o^{r}$ gene. During drug selection $(500 \mu \mathrm{g} / \mathrm{ml}$ ble $)$, drastic changes from normal epimastigote morphology in axenic cultures were observed in these transfectants (Fig. 3B, panel i) but not in wild type parasites, which were cultured in parallel without selective drug (Fig. 3B, panel ii). Amastigote-like forms with short, retracted flagella were abundant ( $>50 \%$ of the culture) among the transfected epimastigote population (Fig. 3B, panel i), whereas the morphology of the parallel wild-type untransfected culture was normal (Fig. 3B, panel ii). Uptake of propidium iodide in these forms indicated that parasite membranes were disrupted (data not shown). These parasites could not be maintained in culture, precluding isolation of gDNA for further genetic analysis.

In summary, this report documents an interesting and selective defect in which organelle duplication (kinetoplast and nuclei) does not appear to be blocked, in which the flagella replicate but in some cases, in which the plasma membranes do not appear to separate effectively. From these studies, it can be surmised that cell division of epimastigotes was impaired selectively. To date, few studies have been published on the mechanism of cell division of T. cruzi (Gomez et al. 1998, 2001, Bogitsh et al. 1999, Grellier et al. 1999a, b, Orr et al. 2000, Santori et al. 2002). The pattern of development, e.g., the time course of DNA replication, organelle duplication and cell division of $T$. cruzi cannot be assumed to occur as has been demonstrated in elegant studies of the related trypanosomatid, T. brucei (Hendriks et al. 2000, McKean 2003). In T. cruzi, targeted disruption of genes for functional analysis remains a challenge and inducible expression systems have not been possible (DaRocha et al. 2004). In addition to the deficit of tools for genetic manipulation of $T$. cruzi, we lack an understanding of the mechanisms regulating T. cruzi gene ex- pression and governing the cellular processes that enable the parasite to transform morphologically. However, recently, the genome sequence of $T$. cruzi CL-Brener strain was completed (El-Sayed et al. 2005). It is anticipated that future developments in transformation, gene disruption and/or inducible expression systems, combined with a comparative genomics approach (Parsons et al. 2005) will enhance our understanding of the complex developmental cycle and morphological transformation required for T. cruzi survival and may identify specific drug targets. Further molecular-genetic studies will be required to evaluate the potential role of the flanking sequences of $G A P D H$, such as promoter functions, in the regulation of cell division and differentiation in T. cruzi.

\section{REFERENCES}

Barrett MP, Burchmore RJ, Stich A, Lazzari JO, Frasch AC, Cazzulo JJ, Krishna S 2003. The trypanosomiases. Lancet 362: 1469-1480.

Bogitsh BJ, Middleton OL, Ribeiro-Rodrigues R 1999. Effects of the antitubulin drug trifluralin on the proliferation and metacyclogenesis of Trypanosoma cruzi epimastigotes. Parasitol Res 85: 475-480.

Burleigh BA, Andrews NW 1998. Signaling and host cell invasion by Trypanosoma cruzi. Curr Opin Microbiol 1: 461465.

DaRocha WD, Otsu K, Teixeira SM, Donelson JE 2004. Tests of cytoplasmic RNA interference (RNAi) and construction of a tetracycline-inducible $\mathrm{T} 7$ promoter system in Trypanosoma cruzi. Mol Biochem Parasitol 133: 175-186.

El-Sayed NM, Myler PJ, Bartholomeu DC, Nilsson D, Aggarwal G, Tran AN, Ghedin E, Worthey EA, Delcher AL, Blandin G, Westenberger SJ, Caler E, Cerqueira GC, Branche C, Haas B, Anupama A, Arner E, Aslund L, Attipoe P, Bontempi E, Bringaud F, Burton P, Cadag E, Campbell DA, Carrington M, Crabtree J, Darban H, da Silveira JF, de Jong P, Edwards K, Englund PT, Fazelina G, Feldblyum T, Ferella M, Frasch AC, Gull K, Horn D, Hou L, Huang Y, Kindlund E, Klingbeil M, Kluge S, Koo H, Lacerda D, Levin MJ, Lorenzi H, Louie T, Machado CR, McCulloch R, McKenna A, Mizuno Y, Mottram JC, Nelson S, Ochaya S, Osoegawa K, Pai G, Parsons M, Pentony M, Pettersson U, Pop M, Ramirez JL, Rinta J, Robertson L, Salzberg SL, Sanchez DO, Seyler A, Sharma R, Shetty J, Simpson AJ, Sisk E, Tammi MT, Tarleton R, Teixeira S, Van Aken S, Vogt C, Ward PN, Wickstead B, Wortman J, White O, Fraser CM, Stuart KD, Andersson B 2005. The genome sequence of Trypanosoma cruzi, etiologic agent of Chagas disease. Science 309: 409-415.

Gomez EB, Kornblihtt AR, Tellez-Inon MT 1998. Cloning of a cdc2-related protein kinase from Trypanosoma cruzi that interacts with mammalian cyclins. Mol Biochem Parasitol 91:337-351.

Gomez EB, Santori MI, Laria S, Engel JC, Swindle J, Eisen H, Szankasi P, Tellez-Inon MT 2001. Characterization of the Trypanosoma cruzi $\mathrm{Cdc} 2 \mathrm{p}$-related protein kinase 1 and identification of three novel associating cyclins. Mol Biochem Parasitol 113: 97-108.

Grellier P, Blum J, Santana J, Bylen E, Mouray E, Sinou V, Teixeira AR, Schrevel J 1999a. Involvement of calyculin A-sensitive phosphatase(s) in the differentiation of Trypanosoma cruzi 
trypomastigotes to amastigotes. Mol Biochem Parasitol 98: 239-252.

Grellier P, Sinou V, Garreau-de Loubresse N, Bylen E, Boulard Y, Schrevel J 1999b. Selective and reversible effects of vinca alkaloids on Trypanosoma cruzi epimastigote forms: blockage of cytokinesis without inhibition of the organelle duplication. Cell Motil Cytoskeleton 42: 36-47.

Hall BF 1993. Trypanosoma cruzi: mechanisms for entry into host cells. Semin Cell Biol 4: 323-333.

Hendriks E, van Deursen FJ, Wilson J, Sarkar M, Timms M, Matthews KR 2000. Life-cycle differentiation in Trypanosoma brucei: molecules and mutants. Biochem Soc Trans 28: 531-536.

Kelly JM, Ward HM, Miles MA, Kendall G 1992. A shuttle vector which facilitates the expression of transfected genes in Trypanosoma cruzi and Leishmania. Nucleic Acids Res 20:3963-3969.

Kendall G, Wilderspin AF, Ashall F, Miles MA, Kelly JM 1990. Trypanosoma cruzi glycosomal glyceraldehyde-3-phosphate dehydrogenase does not conform to the 'hotspot' topogenic signal model. Embo J 9: 2751-2758.

Liu YG, Whittier RF 1995. Thermal asymmetric interlaced PCR: automatable amplification and sequencing of insert end fragments from P1 and YAC clones for chromosome walking. Genomics 25: 674-681.

McKean PG 2003. Coordination of cell cycle and cytokinesis in Trypanosoma brucei. Curr Opin Microbiol 6: 600-607.

Medina-Acosta E, Cross GA 1993. Rapid isolation of DNA from trypanosomatid protozoa using a simple 'mini-prep' procedure. Mol Biochem Parasitol 59: 327-329.
Miles M 2003. American trypanosomiasis (Chagas disease). In G Cook, A Zumla (eds), Manson's Tropical Disease, Elsevier Science, London, p. 1325-1337.

Nozaki T, Cross GA 1995. Effects of 3' untranslated and intergenic regions on gene expression in Trypanosoma cruzi. Mol Biochem Parasitol 75: 55-67.

Orr GA, Werner C, Xu J, Bennett M, Weiss LM, Takvorkan P, Tanowitz HB, Wittner M 2000. Identification of novel serine/ threonine protein phosphatases in Trypanosoma cruzi: a potential role in control of cytokinesis and morphology. Infect Immun 68: 1350-1358.

Parker JD, Rabinovitch PS, Burmer GC 1991. Targeted gene walking polymerase chain reaction. Nucleic Acids Res 19: 3055-3060.

Parsons M, Worthey EA, Ward PN, Mottram JC 2005. Comparative analysis of the kinomes of three pathogenic trypanosomatids: Leishmania major, Trypanosoma brucei and Trypanosoma cruzi. BMC Genomics 6: 127.

Santori MI, Laria S, Gomez EB, Espinosa I, Galanti N, TellezInon MT 2002. Evidence for CRK3 participation in the cell division cycle of Trypanosoma cruzi. Mol Biochem Parasitol 121:225-232.

Stevens JR, Noyes HA, Dover GA, Gibson WC 1999. The ancient and divergent origins of the human pathogenic trypanosomes, Trypanosoma brucei and T. cruzi. Parasitology 118: 107-116.

Zacks MA, Garg N 2006. Recent developments in the molecular, biochemical and functional characterization of GPI8 and the GPI-anchoring mechanism [review]. Mol Membr Biol 23: 209-225. 